\title{
Article
}

\section{Mental health, deprivation, and the neighbourhood social environment: a network analysis}

McElroy, Eoin, Mclntyre, Jason C., Bentall, Richard P., Wilson, Tim, Holt, Keith, Kullu, Cecil, Rajan, Nathan, Kerr, Andrew, Panagaki, Katerina, Mckeown, Mick, Saini, Pooja, Gabbay, Mark and Corcoran, Rhiannon

Available at http://clok.uclan.ac.uk/24943/

McElroy, Eoin, McIntyre, Jason C., Bentall, Richard P., Wilson, Tim, Holt, Keith, Kullu, Cecil, Rajan, Nathan, Kerr, Andrew, Panagaki, Katerina et al (2019) Mental health, deprivation, and the neighbourhood social environment: a network analysis. Clinical Psychological Science, 7 (4). pp. 719-734. ISSN 2167-7026

It is advisable to refer to the publisher's version if you intend to cite from the work. http://dx.doi.org/10.1177/2167702619830640

For more information about UCLan's research in this area go to http://www.uclan.ac.uk/researchgroups/ and search for <name of research Group>.

For information about Research generally at UCLan please go to http://www.uclan.ac.uk/research/

All outputs in CLoK are protected by Intellectual Property Rights law, including Copyright law. Copyright, IPR and Moral Rights for the works on this site are retained by the individual authors and/or other copyright owners. Terms and conditions for use of this material are defined in the policies page. 
Mental health, deprivation, and the neighbourhood social environment: a network analysis

Authors: Eoin McElroy ${ }^{1 *}$, Jason C. McIntyre², Richard P. Bentall ${ }^{3}$, Tim Wilson ${ }^{4}$, Keith Holt $^{4}$, Cecil Kullu ${ }^{5}$, Rajan Nathan ${ }^{6}$, Andrew Kerr ${ }^{7}$, Katerina Panagaki ${ }^{8}$, Mick McKeown ${ }^{9}$, Pooja Saini $^{2}$, Mark Gabbay ${ }^{1}$, Rhiannon Corcoran ${ }^{1}$

${ }^{1}$ Institute of Psychology, Health and Society, University of Liverpool

${ }^{2}$ School of Natural Sciences and Psychology, Liverpool John Moores University

${ }^{3}$ Department of Psychology, University of Sheffield

${ }^{4}$ Collaboration for Applied Health Research and Care - North West Coast

${ }^{5}$ Merseycare NHS Foundation Trust

${ }^{6}$ Cheshire and Wirral Partnership NHS Foundation Trust

${ }^{7}$ NHS Liverpool Clinical Commissioning Group

${ }^{8}$ Spectrum Centre for Mental Health Research, Division of Health Research, Lancaster University

${ }^{9}$ School of Nursing, University of Central Lancashire

*correspondence to: Eoin McElroy, Department of Psychological Sciences, Institute of Psychology, Health and Society, University of Liverpool, 241b Eleanor Rathbone Building, Liverpool, UK. Email: emcelroy@liverpool.ac.uk 
Word count (not including abstract, refs etc.): 9,174

Keywords: deprivation, mental health, neighbourhood environment, network analysis, social capital, antisocial behaviour

Declaration of interest: This work was supported by the National Institute for Health Research, Collaboration for Leadership and Health Research and Care North West Coast (NIHR CLAHRC NWC). The views expressed are those of the authors and not necessarily those of the NHS, the NIHR or the Department of Health. 


\begin{abstract}
Different aspects of the neighbourhood social environment have been linked with mental illhealth, however the mechanisms underlying these associations remain poorly understood because of the number and complexity of the components involved. We used a novel statistical approach, network analysis, to explore the complex associations between neighbourhood social cohesion, social disorder and mental health symptoms in a sample of 3,670 adults from an economically deprived region of the UK (mean age [SD] $=49.34$ years [18.87]; 57\% female). Elasso regularised networks were estimated, and network comparisons were conducted by level of deprivation. Mental health symptoms and neighbourhood components formed relatively distinct clusters of items. These domains were linked primarily by paranoia, although only in the most deprived group. Drunken/rowdy behaviour was particularly influential within the neighbourhood cluster, therefore policies aimed at reducing such disruptive behaviour could have positive knock-on effects for social cohesion and mental health.
\end{abstract}




\section{Introduction}

There is robust evidence that the neighbourhood social environment can influence mental health (Faris \& Dunham, 1939; Peen, Schoevers, Beekman, \& Dekker, 2010; Penkalla \& Kohler, 2014; Sundquist, Frank, \& Sundquist, 2004; Vassos, Pedersen, Murray, Collier, \& Lewis, 2012). This phenomenon is highly complex insofar as multiple components of the neighbourhood social environment likely influence mental health in intricate and potentially cyclical ways. Aspects of the social environment that are more pronounced in deprived neighbourhoods (e.g. low social cohesion and social disorder) have been identified as risk factors for mental ill-health, even after controlling for individual-level risks (Roh, Jang, Chiriboga, Kwag, Cho, \& Bernstein 2011; Wilson-Genderson \& Pruchno, 2013; Stafford, McMunn, \& Roberto De Vogli, 2011). However, the specificity with which these aspects of the neighbourhood influence mental health is poorly understood (Penkalla, \& Kohler, 2014) and there is a continuing need to investigate the effects of physical and social characteristics of deprived living environments on mental distress, especially during times of austerity. Here we report a novel approach to understanding the effects of these characteristics, network analysis, using survey data collected in the North West Coast of England where health and wellbeing inequalities are amongst the highest in the UK (Collins, 2017; Kontopantelis, Mamas, van Marwijk, Ryan, Buchan, Ashcroft, et al., 2017).

\section{Neighbourhood social cohesion}

The need for social connection is a fundamental human drive found across different cultures and contexts (Baumeister \& Leary, 1995). Research suggests that identifying with social groups is associated with lower levels of depression (Cruwys, Haslam, Dingle, Haslam, \& Jetten, 2014; Cruwys, South, Greenaway, \& Haslam, 2015), anxiety (Elahi, McIntyre, Hampson, Bodycote, Sitko, White, et al., 2018; Lee \& Robins, 1998) and paranoia (McIntyre, 
Wickham, Barr, \& Bentall, 2017; Sani, Wakefield, Herrera, \& Zeybek, 2017). Depending on their specific social economic, and physical characteristics, neighbourhoods may either facilitate or impede the formation and maintenance of the kind of social relationships that promote mental health. Neighbourhood social cohesion refers to the levels of connectedness, solidarity, trust and reciprocity amongst a neighbourhood's inhabitants (Kawachi \& Berkman, 2000). Past research suggests that people living in neighbourhoods with high levels of social cohesion are more resilient (Fone, White, Farewell, Kelly, John, Lloyd, et al., 2014) and numerous studies have demonstrated associations between low social cohesion and low mental well-being (Cramm, van Dijk, \& Nieboer, 2013; Gale, Dennison, Cooper, \& Sayer, 2011). However, few studies have looked at the association between cohesion and specific mental health difficulties (Choi \& Matz-Costa, 2018). Those that have done so have focussed primarily on specific psychiatric disorders, e.g. depression (Stafford, McMunn, \& Roberto De Vogli, 2011) or anxiety (Aneshensel \& Sucoff, 1996). However, psychiatric disorders are likely not discrete entities and the symptoms of mental illness are known to be shared across diagnostic boundaries (Borsboom, Cramer, Schmittmann, Epskamp \& Waldorp, 2011), making it difficult to identify specific associations.

\section{Neighbourhood social disorder}

It has been argued that social ties and community belonging alone are not sufficient for maintaining social cohesion. Indeed, social ties need to be grounded in social trust and control, and translated into specific goals for the common good, such as maintaining public order (Sampson, 2003). Neighbourhood social disorder encompasses various forms of antisocial behaviour such as litter, graffiti, crime and other incivilities (Stafford, McMunn, \& Roberto De Vogli, 2011). Several studies have demonstrated an association between social disorder and depression (Wilson-Genderson \& Pruchno, 2013; Booth, Ayers, \& Marsiglia, 
2012; Roh et al., 2011), and it is hypothesised that experiences of social disorder lead to feelings of powerlessness, mistrust and social isolation, which in turn may influence the development/maintenance of depressive symptoms (Booth, et al., 2012). Although social cohesion and social disorder are likely interlinked, few studies have considered these aspects of the neighbourhood social environment in tandem (Choi \& Matz-Costa, 2018). Hence, there is a clear need for studies that investigate the interrelation between neighbourhood cohesion, social disorder, and a range of mental ill-health outcomes.

\section{The neighbourhood social environment: the impact of deprivation}

Empirical studies suggest that neighbourhood environments that are characterised by high levels of deprivation may confer increased risk for mental health difficulties. It seems that even brief or remote exposure to physical and social cues indicative of deprivation are associated with negative perceptions about the self and others (Corcoran, Mansfield, Giokas, Hawkins, Bamford, \& Marshall, 2017). For example, Ellett, Freeman and Garety (2008) explored the acute effects of exposure to a deprived urban environment on the psychology of people experiencing persecutory delusions. They found that brief exposure to a harsh innercity environment was associated with increased feelings of anxiety and persecution as well as an increase in negative beliefs about others. Similarly, Nettle, Pepper, Jobling and Schroeder (2014) compared the level of social trust and paranoia among residents of two neighbourhoods that varied in their level of deprivation. Residents in the deprived neighbourhood displayed significantly lower levels of social trust and significantly higher levels of paranoia compared to the residents of the less deprived neighbourhood, findings that were also true for participants only briefly exposed to the same environments (Nettle et al., 2014). Collectively, these findings suggest that deprived neighbourhood environments may promote paranoid thinking. 
In a recent study by Corcoran, Mansfield, de Bezenac, Anderson, Overbury, and Marshall, (accepted, in press) using experience sampling methodology, it was shown that in situ judgments of neighbourhood trust were strongly predicted by judgements of residents' wealth and by the participants' baseline levels of paranoid ideation. In the same study, level of threat felt by the participants as they walked through a deprived neighbourhood was significantly and independently predicted by self-reported personal resilience of the participants after controlling for other correlates such as family socioeconomic status (SES), weather, depression, anxiety and baseline paranoia. This finding demonstrates the strong relationship between mental distress and place characteristics with Corcoran et al. (accepted, in press) speculating that a tipping point might exist whereby neighbourhoods with too many cues to deprivation may be psychologically intolerable to all but the most resilient.

\section{Mental ill-health and the neighbourhood environment: A Complex Network}

Attempts to unpack the social psychological processes underlying the relationship between the neighbourhood environment and mental health have been hindered by the number and complexity of the components involved, and by the potentially bidirectional and cyclical nature of the interactions between these components. To illustrate, it has been suggested that both the objective and perceived quality of environments contributes to the prevalence of mental distress in certain areas (Ellaway Macintyre, \& Kearns, 2001; Ellaway, Morris, Curtice, Robertson, Allardice, \& Robertson 2009; Gong, Palmer, Gallacher, Marsden, \& Fone, 2016), with disordered and deprived urban neighbourhoods found to be associated with increased perception of threat and hostility, and increased distress in those who live in them (Chen \& Paterson, 2006; Hill, Pollet, \& Nettle, 2014; Ross \& Mirowsky, 2009; Witt, 1989). However, psychological biases associated with depression, anxiety and paranoia (e.g. 
anticipation and avoidance of social threat) may result in more negative reactions to place (Bennett \& Corcoran, 2010; Kellough, Beevers, Ellis, \& Wells, 2008; MacLeod, Mathews, \& Tata, 1986; Mathews, Ridgeway, \& Williamson, 1996; Moutoussis, Williams, Dayan, \& Bentall, 2007). Associations between the neighbourhood environment and mental distress are further complicated by the fact that expressions of mental ill-health are highly heterogeneous (i.e. comprised of multiple symptoms or experiences), and unique relationships may exist between specific symptoms and environmental risk factors (Isvoranu, van Borkulo, Boyette, Wigman, Vinkers, \& Borsboom, 2016). Hence, it has been suggested that traditional analytical approaches, which typically focus their enquiry on broad, latent constructs (e.g. depression, schizophrenia) may struggle to adequately capture the intricacies of such interactions, and alternative modelling strategies should, therefore, be considered (Isvoranu, Borsboom, van Os, \& Guloksuz, 2016).

The network approach to psychopathology is an alternative perspective that may better capture the nuances of neighbourhood-mental health associations. This novel, datadriven approach frames mental health problems as dynamical systems. That is, rather than focus on underlying latent 'disorders', this approach seeks to conceptualise and understand psychopathology as a complex network of locally associated symptoms Such symptomsymptom associations are hypothesised to reflect causal processes linking symptoms (e.g. insomnia $\rightarrow$ fatigue $\rightarrow$ irritability $\rightarrow$ insomnia), shared etiological influences, or a combination of both (Fried \& Cramer, 2017). Network models are typically presented graphically as nodes (points in space that represent variables) and edges (lines that link nodes and reflect the level of association). Strongly connected nodes are placed closer together, and the overall connectivity of nodes (aka centrality) is quantified, which allows us to identify the nodes that are most important to the network as a whole; i.e. the nodes with the most and 
strongest connections. The main advantage of the network approach is that, by focussing on direct associations, we can determine not only how important a node is to the overall network, but also where a node is important within the network (i.e. the strongest edges denoting pathways between symptoms and/or risk factors). Thus, network models may help identify key associations that serve to link different aspects of the neighbourhood environment with mental health symptoms.

\section{The present study}

Network models of psychopathology are increasingly presented in the literature, yet the majority of such studies to date have focussed solely on symptom-symptom associations within disorders (Beard, Millner, Forgeard, Fried, Hsu, Treadway, et al., 2016; Fried, Epskamp, Nesse, Tuerlinckx, \& Borsboom, 2016; McElroy, Fearon, Belsky, Fonagy, \& Patalay, accepted, in press). However, studies are beginning to incorporate environmental variables into psychopathological networks in order to explore different pathways between risk factors and individual symptoms (Isvoranu et al., 2016; Anker, Forbes, Almquist, Menk, Thuras, Unruh, et al., 2017). In this exploratory study, we use network models to examine the associations that exist between a broad range of mental health symptoms and two aspects of the neighbourhood social environment: social cohesion and social disorder. Given the exploratory nature of this study and the methods employed, we make no a-priori predictions about the associations between aspects of the neighbourhood environment and mental health symptoms. Specifically we aim to determine; i) how are mental health symptoms associated with one another as a network and what are the key symptoms within this network, ii) how are social cohesion and social disorder associated as a network, and what components link these two domains, iii) what are the specific bridges that link social cohesion, social disorder and mental health symptoms. Furthermore, in order to explore the potential moderating role 
of neighbourhood deprivation, we compare the network structure of sub-groups that vary by level of deprivation. We hypothesise that there will be more and/or stronger connections between the neighbourhood social environment and mental health in the more deprived groups. 


\section{Method}

\section{Sample}

We surveyed 4319 people from the North West Coast of England using face-to-face interviews between August 2015 and January 2016. The sample was drawn from an area of high national deprivation; i.e. the north west of England. Within this economically disadvantaged population, a random probability sample was taken from 20 high deprivation areas, and 8 low-deprivation areas. Each area had a population of approximately 10,000 people and the majority of areas were defined by electoral ward boundaries. The areas were selected by local authority representatives based on the following considerations: population size (5,000-10,000 people), level of disadvantage (as measured via Index of Multiple Deprivation), coherent shared identity, and infrastructure for policy delivery. One adult participant was interviewed per household (McIntyre et al., 2017). Fifty-seven per cent of the sample identified as female, and ages ranged from 18 to $95(\mathrm{M}=49.12, \mathrm{SD}=19.13)$. The majority of the sample (89\%) reported having white ethnic backgrounds. How best to address missing data in network analysis is currently debated (Santos, Fried, Asafu-Adjei, \& Ruiz, 2017), therefore the present study used the most common current practice: listwise deletion (Fried et al., 2016; Isvoranu et al., 2016; Santos et al., 2017). Complete data were available for 3,670 participants, and analyses were conducted using this sub-sample. Of those who were included in the study, the mean age was 49.34 years $(\mathrm{SD}=18.87), 57 \%$ were female, $89 \%$ described their ethnicity as white, and had a mean index of multiple deprivation (IMD) score of $39.65(\mathrm{SD}=21.05)$. Those who were excluded due to missing data were more likely to be white British $\left(\chi^{2}(1)=4.10, \mathrm{p}=0.04\right)$ and scored higher on the index of multiple deprivation $(\mathrm{t}(4,317)=-6.18), \mathrm{p}=0.04)$, but did not differ in terms of age $(\mathrm{t}(835.63)=1.58)$, $\mathrm{p}=0.12)$, or gender $\left(\chi^{2}(1)=3.45, \mathrm{p}=0.06\right)$. 


\section{Measures}

\section{Depression}

Depression was assessed with the nine-item Patient Health Questionnaire (PHQ-9; Kroenke \& Spitzer, 2002) . Participants reported how often they had been bothered by problems such as "little interest or pleasure in doing things" and "feeling down, depressed, or hopeless" on a scale from $0=$ not at all to $3=$ nearly every day.

\section{Anxiety}

We administered the seven item Generalised Anxiety Disorder Scale (GAD-7; Spitzer, Kroemke, Williams, \& Lowe, 2006) which assesses frequency of events such as "worrying too much about different things" and "trouble relaxing" over the previous two weeks on a four-point scale $(0=$ not at all, $3=$ nearly every day $)$.

\section{Paranoia}

Paranoia was assessed using a short form of the Persecution and Deservedness Scale (PaDS; Melo, Corcoran, Shryane, \& Bentall, 2009). This five item version of the PaDS has been found to be highly (.94) correlated with the full PaDS, and taxometric analyses have shown that the reduced scale measures a continuum of paranoid thinking running from healthy functioning through mild paranoia to clinical psychosis (Elahi et al., 2017). Participants rated their level of agreement with statements such as 'I'm often suspicious of other people's intentions towards me" on a five-point scale $(0=$ strongly disagree, $4=$ strongly agree $)$.

\section{Auditory Verbal Hallucinations (AVH)}

AVH was measured with two items adapted from the Launay-Slade Hallucination Scale (Launay \& Slade, 1981): "I have been troubled by hearing voices in my head" and "I often 
hear a voice speaking my thoughts aloud." Participants rated their level of agreement with each statement on a five-point scale ranging from $0=$ strongly disagree, $4=$ strongly agree .

\section{Neighbourhood Social Environment}

Ten self-report items taken from a survey administered by the UK Office for National Statistics (Harper \& Kelly, 2003) were used to measure different aspects of the neighbourhood social environment. These items covered both social cohesion (sense of neighbourhood belonging, trust of neighbours, neighbours looking out for one another, ability to influence local decisions) and social disorder (drunkenness /rowdiness, littering, vandalism, harassment of ethnic minorities, teenagers loitering, and troublesome neighbours). Responses were indicated on Likert-type scales (see Table S1 online for specific item content).

\section{Social Connectivity}

In order to control for potential protective effects of relationships outside of a neighbourhood context (e.g. friends, family), general social connectivity was assessed using two questions from the 2013-14 Community Life Survey (UK Cabinet Office, 2014). We asked participants the extent to which they agreed with the statements: "If I wanted company or to socialise, there are people I can call on" and "If I needed help, there are people who would be there for me". Response options for these two items ranged from $1=$ definitely agree to $4=$ definitely disagree. In addition, participants were asked to report the number of people they lived with (dichotomised as $0=$ lives with someone else, $1=$ lives alone).

\section{Deprivation}


Deprivation was measured using the Index of Multiple deprivation (IMD) (UK Department for Communities and Local Government, 2015). The IMD is based on 37 separate indicators, reflecting seven different domains of deprivation which are combined, using appropriate weights to form an overall score (UK Department for Communities and Local Government, 2015). Participants were divided into three groups based on their IMD scores; lowest deprivation (lowest $33 \% ; n=1310$ ), moderate deprivation (middle 33\%; $n=1192$ ), highest deprivation (highest 33\%; $\mathrm{n}=1168$ ).

\section{Data preparation}

Network methodologies that deal with continuous data assume that variables are normally distributed, which is unlikely to be the case in community mental health data (van Borkulo, Borsboom, Epskamp, Blanken, Boschloo, Schoevers, et al., 2014). Binary network methodologies do not make this assumption (van Borkulo et al., 2014), and given that the data were a mixture of binary and ordinal variables, ordinal items were dichotomised (for full details see online supplementary materials, Table S1).

\section{Statistical analysis}

\section{Elasso Regularised Networks}

Networks were estimated using the R package, 'Isingfit' (van Borkulo et al., 2014), which was developed to construct weighted undirected networks using binary variables. This package employs elasso, a methodology based on the Ising model (Ising, 1925), in which each variable is regressed on all other variables with a lasso $\left(l_{1}\right)$ penalty employed to shrink regression coefficients and set very small coefficients to zero (van Borkulo \& Epskamp, 2014). The degree of shrinkage is determined using the extended Bayesian Information Criterion (EBIC; Epskamp \& Fried, 2016). This method identifies the optimal network 
structure, striking a balance between parsimony and goodness of fit (van Borkulo \& Epskamp, 2014). 'Isingfit' produces undirected edges that are the mean values of the two logistic regression coefficients (i.e. node A predicting node B, and node B predicting node A), which can be interpreted similarly to partial correlations (i.e. an edge/line linking two nodes reflects a statistically significant association controlling for all other nodes in the network). Networks were graphically illustrated using the 'qgraph' package (Epskamp et al., 2012), which implements the Fruchterman-Reingold algorithm to place highly connected nodes closer together (Fruchterman \& Reingold, 1991). In total, four networks were constructed. First a network of 36 binary nodes was constructed using the overall sample $(\mathrm{N}=3670)$. Next, three separate networks were constructed for each deprivation group. Community structures (i.e. clusters of nodes nested within the overall network) were identified using the walktrap algorithm (Pons \& Latapy, 2006) available in the 'igraph' package (Csardi \& Nepusz, 2006).

\section{Centrality}

In order to identify the nodes that were most important to the networks, three common measures of node centrality were calculated. Degree strength is determined by summing the standardised weights of all significant edges in the network (Costantini, Epskamp, Borsboom, Perugini, Mõttus, Waldorp, et al., 2015). Closeness is calculated by taking the inverse of the sum of distances of the node of interest from all other nodes in a given network (Opsahl, Agneessens, \& Skvoretz, 2010). Betweenness sums the number of times each node lies on the shortest path between two other nodes. Centrality indices are presented as standardized zscores, with higher values indicative of greater importance to the network as a whole (Costantini et al., 2015). 
In order to identify potential bridging nodes (i.e. nodes that are integral in connecting different clusters), we calculated bridge centrality values using the 'networktools' package (Jones, 2017). Bridge centrality values are calculated in the same way as the node centrality metrics described above, but focus exclusively on the connections between a node of interest and nodes in different clusters (thus quantifying how important a given node is in linking clusters). For instance to calculate the bridge strength of item \#1 from the PHQ-9, we would sum the strength of connections that this item has with all other items in the network, excluding the other eight PHQ-9 items. Bridge centrality indices are also presented as standardized z-scores; with higher values indicating that nodes are more important for bridging clusters.

\section{Accuracy/reliability of networks}

Edge weight accuracy and centrality stability refer to the degrees of confidence with which one can interpret the rank ordering of the edge weights and centrality values (strongest to weakest), and these were assessed using the 'Bootnet' package in R (Epskamp, Borsboom, \& Fried, 2017). Bootstrapped difference tests and case-dropping sub-sampling procedures were performed following the guidelines of Epskamp, Borsboom, and Fried (2017). Detailed guidelines of these procedures are presented in the online supplementary materials (S1).

\section{Network comparisons based on deprivation level}

To test for structural changes (i.e. changes in the relationships between individual nodes) and changes in overall connectivity (i.e. the summed weights of the edges) based on deprivation level (low, moderate, high), networks were compared using the 'NetworkComparisonTest' (NCT) package in R (van Borkulo et al., 2016). The NCT procedure involves non-parametric 
permutation testing. A detailed description of this process is available in the online supplementary materials (S2). 


\section{Results}

\section{Descriptive statistics}

Item-level (Table S1) and scale-level (Table S2) descriptive statistics are presented in the online supplementary materials. Based on established cut-offs $(M \geq 6)$ (Spitzer et al., 2006), $1055(29 \%)$ participants reported at least moderate levels of depression and $828(23 \%)$ met the criteria for moderate general anxiety

\section{Elasso Regularised Networks}

The elasso regularised network for the full sample $(\mathrm{N}=3,670)$ is presented in Figure 1 . The network was highly interconnected; out of a potential 666 edges, $480(72 \%)$ were estimated to be above zero. The bootstrapped difference test indicated that the rank ordering of edge weights (i.e. thickness of edges) could be interpreted confidence (Figure S1). Overall, the strongest edges were 'Has people to call for help - Has people to call for company', followed by 'Hearing voices - Thoughts broadcast out loud', and 'Worrying too much - Worrying about different things'. The case-dropping subset bootstrap method (Figure S2) indicated that only the strength values were reliable $(C S$ coefficient $=0.75)$, therefore we will focus solely on this metric (Figure 2). 'Worrying too much' had the highest strength value, indicating that it had strong direct associations with other nodes in the network. 'Trouble relaxing', and 'Concentration problems', were also high in strength. 'People drunk/rowdy in public' had the highest strength of the neighbourhood variables.

<insert Figure 1 around here>

\section{Associations within mental health symptoms and within neighbourhood social characteristics}

As shown in Figure 1, obvious clusters reflecting depression, general anxiety, paranoia and auditory hallucinations emerged, with all items positively associated. The distinction of these 
clusters was supported by the walktrap algorithm (Figure S3). These clusters, however, were highly interconnected, with multiple positive associations crossing diagnostic boundaries. Edges between anxiety and depression items were particularly frequent and strong. The nodes 'Feeling nervous, anxious or on edge' and 'Concentration problems' were high in bridge strength (Figure 3), indicating they were particularly strongly associated with nodes in other mental health clusters. The auditory hallucination symptoms were connected to the rest of the network via paranoia and depressive items.

$$
\begin{aligned}
& \text { <insert Figure } 2 \text { around here> } \\
& \text { <insert Figure } 3 \text { around here> }
\end{aligned}
$$

With regards to the neighbourhood items, two clusters reflecting social cohesion and social disorder emerged. As could be expected, the associations between the two clusters were negative, with high cohesion associated with low disorder. The social disorder node 'People drunk/rowdy in public' had high bridge strength (Figure 3), indicating that it was particularly strongly (and negatively) associated with social cohesion.

\section{Associations between mental health symptoms and neighbourhood social characteristics}

With regards to links between the neighbourhood social environment and mental health, paranoia appeared to have an important bridging role, with 6 significant edges (two positive with social disorder items and four negative with social cohesion items) between these two broad domains. 'Suspicious of other people's intentions' and 'Feels that people mean harm' were moderately high in bridge strength, suggesting they may act as key bridges between these two broad domains. Neighbourhood cohesion was also directly and negatively linked with anxiety ('Neighbours look out for each other' - 'Worrying too much about different things'), and the social disorder node 'Teenagers hanging around' was directly linked to 'Easily annoyed or irritable'. Surprisingly perhaps, the neighbourhood social environment 
was not directly associated with depression nodes, suggesting that the impact of the social environment on mood is mediated by more direct influences on anxiety and paranoia. Less surprisingly neighbourhood characteristics were also not directly associated with hallucination nodes. Finally, even though it was included primarily for control purposes, social connectivity items demonstrated no unique associations with any of the mental health items, but were associated with the node 'Neighbours look out for each other'.

\section{Comparison by deprivation}

The sample was spilt by deprivation level (IMD tertiles; Figure 4). The centrality values for these networks are presented in the online supplementary materials (Figure S4-S6). <insert Figure 4 around here>

Results from the non-parametric permutation tests, demonstrated that there were no significant differences in global strength values (i.e., overall connectivity of items) between the three networks (Figure S7). However, the low-deprivation and high-deprivation networks demonstrated a significant difference in structure (Figure S7). As such, while the overall strength of associations were of a similar magnitude across the deprivation groups, the manner in which the items were connected differed between low and high deprivation groups. As predicted, the high deprivation group had a greater number of significant edges both within and across the domains of mental health and neighbourhood environment. Notably, there were no significant bridges between the neighbourhood nodes and mental health nodes for the low-deprivation group, and only one bridge for the mid-deprivation groups, whereas in the high deprivation group there were three bridges between these domains, and again these involved social cohesion and paranoia. 


\section{Discussion}

This is the first study to use the network framework to explore the relationship between mental ill health and the neighbourhood social environment. This novel approach allowed us to capture and quantify the complex associations that exist both within and across these two broad domains. Our analyses demonstrated equifinal and multifinal pathways between specific components of the neighbourhood social environment and mental health symptoms.

\section{Mental health symptoms as a network}

Although the mental health symptoms in the present network formed clusters corresponding to their diagnostic constructs, these clusters were highly interlinked. In particular, there were many significant associations between individual symptoms of depression and anxiety, a finding that is in line with recent studies that have highlighted the overlap between these disorder constructs (Bekhuis et al., 2016; Fried et al., 2016; McElroy et al., accepted, in press; Lamers van Oppen, Smit, Spinhoven, van Balkom, Nolen, 2011). An examination of bridge strength demonstrated that 'Feeling nervous, anxious or on edge' and 'Concentration problems' had strong connections with nodes in different clusters, suggesting they are influential symptoms in linking the four mental health domains. The interconnected nature of the four mental health symptom clusters is in line with previous network studies that have explored broad patterns of comorbidity (McElroy \& Patalay, under review; Boschloo, Schoevers, van Borkulo, Borsboom \& Oldehinkel, 2016). Collectively, these findings challenge the sharpness of our diagnostic boundaries, and it is thus unsurprising that comorbidity is the rule rather than the exception when strict categorical diagnoses are used in research and/or clinical practice. Psychopathology may therefore be better conceived as a dynamical system of directly associated symptoms (Borsboom et al., 2013). 


\section{The neighbourhood social environment as a network}

Similarly, items pertaining to the neighbourhood social environment formed two distinct, yet interrelated clusters reflecting social cohesion and social disorder. The nodes 'People drunk/rowdy in public' and 'Troublesome neighbours' were notably high in bridge strength, and they had particularly strong (and negative) ties to social cohesion. This is in line with previous research that has demonstrated that the upkeep of public order is key in fostering and maintaining a sense of social cohesion (Sampson, 2003). The frequency and strength of connections between these clusters demonstrate that the neighbourhood social environment, much like mental health, may be conceptualised as a dynamical system of mutually associated elements.

\section{Bridges between the neighbourhood social environment and mental health}

Although neighbourhood and mental health items appeared to form two broad clusters of nodes, a total of eight significant edges were observed between these two domains. These edges suggest different pathways, both direct and indirect, between components of the neighbourhood social environment and mental health symptoms. Notably, symptoms of paranoia played a strong role in bridging these two broad domains. Although traditionally viewed as an indicator of severe mental illness (i.e. psychotic disorder), recent evidence has suggested that paranoia is better conceptualised as an exponentially distributed, continuum within the general population (Elahi, Algorta, Varese, McIntyre, \& Bentall, 2017; Bebbington, McBride, Steel, Kuipers, Radovanoviĉ, Brugha, et al., 2013). In other words, it is relatively common for people to be wary of the intentions of others, and these feelings only become clinically relevant when they result in excessive amounts of distress or impaired functionality. In such cases, more severe persecutory delusions are also more likely to emerge (Bebbington et al., 2013). Of the five symptoms of paranoia, 'Suspicious of other people's 
intentions' had both the highest strength and bridge strength, suggesting that it served as a nexus between the neighbourhood social environment and other forms of psychopathology, including more severe persecutory delusions. Although the direction of effect cannot be established in this undirected network, it is plausible that the associations between paranoia and the perceived neighbourhood environment are bidirectional. In other words, a negative neighbourhood social environment may lead to increased perceptions of threat and hostility (Chen \& Paterson, 2006; Hill et al., 2014; Ross \& Mirowsky, 2009; Witt, 1989), whereas existing mental health difficulties may predispose individuals to negative evaluations of place characteristics (Bennett \& Corcoran, 2010; Kellough et al., 2008; MacLeod et al., 1986; Mathews et al., 1996; Moutoussis et al., 2007). As such, the relationship between the neighbourhood social environment and mental health may best be described as a dynamical system, with common symptoms of paranoia acting as key intersections.

Further examination of the edges between neighbourhood and mental health nodes revealed an additional interesting pattern. Neighbourhood disorder nodes that involve overtly hostile/threatening behaviour (e.g. drunken/rowdy behaviour, teenagers loitering) were directly associated with anxiety and paranoia items, supporting a plausible pathway whereby increased threat from others may feed into and reinforce the anticipation and avoidance of social threat, which is a common facet of mental health problems (Freeman, Gittins, Pugh, Antley, Slater, \& Dunn, 2008; Van Os, Linscott, Myin-Germeys, Delespaul, \& Krabbendam et al., 2009). Conversely, physical and social environmental cues relating to social disorder (e.g. vandalism and litter) were not associated with mental health symptoms directly, but rather were indirectly associated with mental health symptoms through social cohesion items (e.g. neighbourhood belonging). This indicates both that interpersonal threat is an important direct influence on paranoid beliefs whereas the influence of physical aspects of the 
neighbourhood environment on mental health symptoms is mediated by a perceived lack of social cohesion. This supports previous research conducted by Nettle et al. (2014), which reported that levels of social trust were lower, and levels of paranoia higher, in deprived neighbourhoods, even among participants who were only briefly exposed to these environments. Collectively, these findings suggest a potential pathway, whereby harsh observable aspects of the neighbourhood environment (e.g. excessive litter, visible vandalism) impact on perceptions of the area (i.e. low social cohesion), which in turn lead to paranoid ideation (Corcoran et al., 2017).

Another notable finding was the lack of direct associations between neighbourhood nodes and auditory hallucinations. This suggests that the effects of neighbourhood environmental factors on such hallucination are mediated by symptoms of paranoia and anxiety. A recent analysis of data from the UK Adult Psychiatric Morbidity Survey, using methods that could estimate direction of causality, similarly found that hallucinations were likely a consequence of paranoia, rather than vice-versa (Moffa, Catone, Kuipers, Kuipers, Freeman, Marwaha, et al., 2017) and van Rooijen, Isvoranu, Meijer, van Borkulo, Ruhé, and de Haan (2017), using a network approach on symptom data from patients, found that delusional beliefs mediated the relationship between hallucinations and other types of psychopathology. In a study by Isvoranu et al. (2016) using the network framework, symptoms of general psychopathology mediated the associations between different types of childhood trauma and psychotic symptoms. Collectively, these findings suggest an affective pathway, whereby environmental risk factors impact psychotic symptoms via more common forms of psychopathology. Together, these findings highlight the ability of network analysis to uncover complex interactions that may be missed by traditional analytical approaches. By contrast, Wickham, Taylor, Shevlin, and Bentall (2014) found that social deprivation was 
positively associated with paranoia, but not auditory hallucinations, concluding that living in a deprived urban area was not a risk factor for the development of hallucinations. However, by taking a dynamical system viewpoint, the findings of the present study suggest a more nuanced pathway; i.e. aspects of the neighbourhood social environment may influence hallucinations indirectly through symptoms of paranoia and anxiety.

\section{Mental health and the neighbourhood: the role of deprivation}

The above effects were further explored by comparing networks based on deprivation (assessed using the IMD). The network structures differed for those in the lowest and highest thirds in terms of overall deprivation. Notably, there were no significant edges between neighbourhood and mental health items in the low deprivation group, whereas for the high deprivation group, a number of significant edges were observed between social cohesion and symptoms of paranoia. It therefore appears that the effect of the neighbourhood environment on mental health operates as a function of overall deprivation, with associations between neighbourhood components and mental health symptoms becoming increasingly relevant as deprivation increases. This finding supports the idea of a 'tipping point' whereby neighbourhood environments that accrue too many negative characteristics (e.g. social disorder, low cohesion) begin to impact on the mental health of inhabitants (Corcoran et al., accepted, in press). The identification of where this tipping point might be and of how neighbourhood characteristics and notions of social cohesion are emotionally weighted to determine their contribution to overall environmental stress (shown here in expressions of anxiety and paranoia) are clear areas for further research.

\section{Limitations, implications and future directions}


The present study is novel in that it is the first to consider the relationship between the neighbourhood social environment and mental health from a dynamical systems perspective. As with all cross-sectional studies, causality cannot be established (Epskamp, van Borkulo, van der Veen, Servaas, Isvoranu, Riese, et al., 2018). However, the aim of this study was not to infer strict causation, but to use network analysis to explore the complex associations that exist between mental health symptoms and the neighbourhood social environment, and to identify key bridging nodes and edges within these networks.

With regards to limitations, although the present study grouped participants based on their relative levels of deprivation, the overall sample was drawn from an economically deprived area of the UK, and thus findings may not be generalizable to more advantaged areas, or areas with marked social/economic inequality. Second, in any given network, the absence of an edge indicates one of two possibilities: i) the edge does not exist (i.e. the two nodes are not associated after controlling for all other nodes in the network) or ii) there is insufficient power for the edge to be detected (Epskamp et al., 2017). Although statistical power remains an under-researched aspect of network analysis (Epskamp et al., 2017), and the sample used in the present study was reasonably large, greater statistical power may have uncovered further associations between neighbourhood and mental health variables. On a related note, although the process of dichotomising ordinal items was justified given the non-normal distribution of items, this process likely resulted in some loss of information and reduced statistical power (Altman \& Royston, 2004). Finally, it is worth noting that the present study used data from a general population sample, therefore it is unclear whether the associations identified herein generalise to individuals with clinical levels of mental health difficulties. 
Bearing the above limitations in mind, the findings of this study have implications for research and broader policy. In terms of research, particularly in the area of psychiatric comorbidity, the symptoms that were highest in strength and bridge strengths (i.e. those that were most influential in connecting the network) reflected common experiences of emotional distress/negative affect, e.g. worry, concentration problems, 'feeling on edge'. Similar findings have been reported in previous network studies (Borsboom et al., 2011), and suggest that these experiences cut-across diagnostic borders, and therefore may account for comorbidity between categorical diagnoses, and the correlations observed between broader transdiagnostic dimensions (Caspi, Houts, Belsky, Goldman-Mellor, Harrington, Israel, et al., 2014).

In terms of broader policy, two key takeaway findings emerged. First, links between the neighbourhood social environment and mental health only emerged in the most deprived third of participants. Thus, socially deprived areas should take precedence in any neighbourhood-level policies designed to improve mental health. Second, general social disruption (e.g. drunkenness/rowdiness in public) was the most highly influential facet of the neighbourhood social environment in the present network, and was linked both directly and indirectly (via low social cohesion) to mental health difficulties. As such, policies aimed at reducing neighbourhood-wide social disorder could have knock-on effects, improving neighbourhood social cohesion which in turn could have positive benefits for the mental health and wellbeing of residents.

\section{Conclusions}

In conclusion, this is the first study to conceptualise and model the interplay between the neighbourhood social environment and mental health as a network of interacting components and symptoms. Neighbourhood and mental health nodes formed two broad clusters, and nested 
within these domains were groups of items reflecting social cohesion, social disorder, depression, anxiety paranoia and auditory hallucinations. Common symptoms of anxiety (e.g. worry, feeling on edge) and depression (concentration problems) were key in linking the mental health portion of the network, whereas drunken/disruptive behaviour was highly influential in linking neighbourhood disorder and cohesion. Symptoms of paranoia, in particular suspicions regarding the intentions of others, served to link the two broad domains of mental health and the neighbourhood environment. Moreover, the links between the mental health and the neighbourhood social environment were only observed in cases of high deprivation. As such, policies aimed at reducing social disruption, particularly in deprived areas, may have positive implications on mental health by way of increasing social cohesion. Overall, our analyses illustrate the complex mechanisms by which the neighbourhood social environment interacts with mental health symptoms. 


\section{References}

Altman, D.G., \& Royston, P. (2006). The cost of dichotomising continuous variables. BMJ, $332,1080$.

Aneshensel, C. S., \& Sucoff, C. A. (1996). The neighborhood context of adolescent mental health. Journal of Health and Social Behavior, 37, 293-310.

Anker, J. J., Forbes, M. K., Almquist, Z. W., Menk, J. S., Thuras, P., Unruh, A. S., \& Kushner, M. G. (2017). A network approach to modeling comorbid internalizing and alcohol use disorders. Journal of Abnormal Psychology, 126, 325-339.

Baumeister, R.F., \& Leary, M.R. (1995). The need to belong: desire for interpersonal attachments as a fundamental human motivation. Psychological Bulletin, 117, 497529.

Beard, C., Millner, A., Forgeard, M., Fried, E., Hsu, K., Treadway, M., Leonard, C. V., Kertz, S. J., \& Björgvinsson, T. (2016). Network analysis of depression and anxiety symptom relationships in a psychiatric sample. Psychological Medicine, 46, 33593369.

Bebbington, P. E., McBride, O., Steel, C., Kuipers, E., Radovanoviĉ, M., Brugha, T., ... \& Freeman, D. (2013). The structure of paranoia in the general population. The British Journal of Psychiatry, 202, 419-427.

Bekhuis, E., Schoevers, R., van Borkulo, C., Rosmalen, J., \& Boschloo, L. (2016). The network structure of major depressive disorder, generalized anxiety disorder and somatic symptomatology. Psychological Medicine, 46, 2989-2998.

Bennett, K., \& Corcoran, R. (2010). Biases in everyday reasoning: Associations with subclinical anxiety, depression and paranoia. Psychosis, 2, 227-237. 
Booth, J., Ayers, S. L., \& Marsiglia, F. F. (2012). Perceived neighborhood safety and psychological distress: Exploring protective factors. Journal of Sociology and Social Welfare, 39, 137-156.

Borsboom, D., \& Cramer, A.O. (2013). Network analysis: an integrative approach to the structure of psychopathology. Annual Review of Clinical Psychology, 9, 91-121.

Borsboom, D., Cramer, A. O., Schmittmann, V. D., Epskamp, S., \& Waldorp, L. J. (2011). The small world of psychopathology. PloS One, 6, e27407.

Boschloo, L., Schoevers, R. A., van Borkulo, C. D., Borsboom, D., \& Oldehinkel, A. J. (2016). The network structure of psychopathology in a community sample of preadolescents. Journal of Abnormal Psychology, 125, 599.

Caspi, A., Houts, R. M., Belsky, D. W., Goldman-Mellor, S. J., Harrington, H., Israel, S., ... \& Moffitt, T. E. (2014). The p factor: One general psychopathology factor in the structure of psychiatric disorders?. Clinical Psychological Science, 2, 119-137.

Chen, E., \& Paterson, L.Q. (2006). Neighborhood, family, and subjective socioeconomic status: How do they relate to adolescent health? Health Psychology, 25, 704.

Choi, Y. J., \& Matz-Costa, C. (2017). Perceived neighborhood safety, social cohesion, and psychological health of older adults. The Gerontologist, 58, 196-206.

Collins, B. (2017). Results from a Well-Being Survey in the North West of England: Inequalities in EQ-5D-Derived Quality-Adjusted Life Expectancy Are Mainly Driven by Pain and Mental Health. Value in Health, 20, 174-177.

Corcoran, R., Mansfield, R., de Bezenac, C., Anderson, E., Overbury, K., \& Marshall, G. (manuscript accepted). Perceived neighbourhood affluence, mental health and wellbeing influence judgements of threat and trust on our streets: an urban walking study. PloS One. 
Corcoran, R., Mansfield, R., Giokas, T., Hawkins, A., Bamford, L., \& Marshall, G. (2017). Places Change Minds: Exploring the Psychology of Urbanicity Using a Brief Contemplation Method. SAGE Open, 7, 2158244017707004.

Costantini, G., Epskamp, S., Borsboom, D., Perugini, M., Mõttus, R., Waldorp, L. J., \& Cramer, A. O. (2015). State of the aRt personality research: A tutorial on network analysis of personality data in R. Journal of Research in Personality, 54, 13-29.

Cramm, J. M., van Dijk, H. M., \& Nieboer, A. P. (2013). Het belang van sociale cohesie en sociaal kapitaal in de buurt voor het welzijn van ouderen. Tijdschrift voor gerontologie en geriatrie, $44,50-58$.

Cruwys, T., Haslam, S.A., Dingle, G.A., Haslam, C., \& Jetten, J. (2014). Depression and social identity: An integrative review. Personality and Social Psychology Review, 18, $215-238$

Cruwys, T., South, E.I., Greenaway, K.H., \& Haslam, S.A. (2015). Social identity reduces depression by fostering positive attributions. Social Psychological and Personality Science, 6, 65-74.

Csardi, G., \& Nepusz, T. (2006). The igraph software package for complex network research. InterJournal, Complex Systems, 1695, 1-9.

Elahi, A., McIntyre, J. C., Hampson, C., Bodycote, H. J., Sitko, K., White, R. G., \& Bentall, R. P. (2018). Home is where you hang your hat: host town identity, but not hometown identity, protects against mental health symptoms associated with financial stress. Journal of Social and Clinical Psychology, 37, 159-181.

Elahi, A., Algorta, G.P., Varese, F., McIntyre, J., \& Bentall, R. (2017). Do paranoid delusions exist on a continuum with subclinical paranoia? A multi-method taxometric study. Schizophrenia Research, 190, 77-81. 
Ellaway, A., Macintyre, S., \& Kearns, A. (2001). Perceptions of place and health in socially contrasting neighbourhoods. Urban Studies, 38, 2299-2316.

Ellaway, A., Morris, G., Curtice, J., Robertson, C., Allardice, G., \& Robertson, R. (2009). Associations between health and different types of environmental incivility: a Scotland-wide study. Public Health, 123, 708-713.

Ellett, L., Freeman, D., \& Garety, P.A. (2008). The psychological effect of an urban environment on individuals with persecutory delusions: The Camberwell walk study. Schizophrenia Research, 99, 77-84.

Epskamp, S., Borsboom, D., \& Fried, E.I. (2017). Estimating psychological networks and their accuracy: a tutorial paper. Behavior Research Methods, 50, 195-212.

Epskamp, S., Cramer, A.O., Waldorp, L.J., Schmittmann, V.D., \& Borsboom, D. (2012). qgraph: Network visualizations of relationships in psychometric data. Journal of Statistical Software, 48, 1-18.

Epskamp, S., \& Fried, E.I. (2016). A Primer on estimating regularised psychological networks. arXiv preprint arXiv:1607.01367.

Epskamp, S., van Borkulo, C. D., van der Veen, D. C., Servaas, M. N., Isvoranu, A. M., Riese, H., \& Cramer, A. O. (2018). Personalized network modeling in psychopathology: The importance of contemporaneous and temporal connections. Clinical Psychological Science, 6, 416-427.

Faris, R. E. L., \& Dunham, H. W. (1939). Mental disorders in urban areas: an ecological study of schizophrenia and other psychoses. Oxford, England: Univ. Chicago Press.

Fone, D., White, J., Farewell, D., Kelly, M., John, G., Lloyd, K., Williams, G., \& Dunstan, F. (2014). Effect of neighbourhood deprivation and social cohesion on mental health inequality: a multilevel population-based longitudinal study. Psychological Medicine, 44, 2449-2460. 
Freeman, D., Gittins, M., Pugh, K., Antley, A., Slater, M., \& Dunn, G. (2008). What makes one person paranoid and another person anxious? The differential prediction of social anxiety and persecutory ideation in an experimental situation. Psychological Medicine, 38, 1121-1132.

Fried, E.I., \& Cramer, A.O. (2017). Moving forward: challenges and directions for psychopathological network theory and methodology. Perspectives on Psychological Science, 12, 999-1020.

Fried, E.I., Epskamp, S., Nesse, R.M., Tuerlinckx, F., \& Borsboom, D. (2016). What are'good'depression symptoms? Comparing the centrality of DSM and non-DSM symptoms of depression in a network analysis. Journal of Affective Disorders, 189, 314-320.

Fruchterman, T.M., \& Reingold, E.M. (1991). Graph drawing by force-directed placement. Software: Practice and Experience, 21, 1129-1164.

Gale, C. R., Dennison, E. M., Cooper, C., \& Sayer, A. A. (2011). Neighbourhood environment and positive mental health in older people: the Hertfordshire Cohort Study. Health \& Place, 17, 867-874.

Gong, Y., Palmer, S., Gallacher, J., Marsden, T., \& Fone, D. (2016). A systematic review of the relationship between objective measurements of the urban environment and psychological distress. Environment International, 96, 48-57.

Harper, R., \& Kelly, M. (2003). Measuring social capital in the United Kingdom. London, Office for National Statistics.

Hill, J., Pollet, T.V., \& Nettle, D. (2014). Disorder affects judgements about a neighbourhood: police presence does not. PeerJ, 2, e287.

Ising, E. (1925). Beitrag zur theorie des ferromagnetismus. Zeitschrift für Physik A Hadrons and Nuclei, 31, 253-258. 
Isvoranu, A.-M., Borsboom, D., van Os, J., \& Guloksuz, S. (2016). A network approach to environmental impact in psychotic disorder: Brief theoretical framework. Schizophrenia Bulletin, 42, 870-873.

Isvoranu, A.-M., van Borkulo, C.D., Boyette, L.-L., Wigman, J.T., Vinkers, C.H., Borsboom, D., et al. (2016). A network approach to psychosis: Pathways between childhood trauma and psychotic symptoms. Schizophrenia Bulletin, 43, 187-196.

Jones, P. J. (2017). Networktools: Tools for Identifying Important Nodes in Networks. R Package Version 1.1. 0.

Kawachi, I., \& Berkman, L. (2000). Social cohesion, social capital, and health. Social Epidemiology, 174, 190.

Kellough, J.L., Beevers, C.G., Ellis, A.J., \& Wells, T.T. (2008). Time course of selective attention in clinically depressed young adults: An eye tracking study. Behaviour Research and Therapy, 46, 1238-1243.

Kontopantelis, E., Mamas, M. A., van Marwijk, H., Ryan, A. M., Buchan, I. E., Ashcroft, D. M., \& Doran, T. (2017). Geographical epidemiology of health and overall deprivation in England, its changes and persistence from 2004 to 2015: A longitudinal spatial population study. Journal of Epidemiology and Community Health, jech-2017209999.

Kroenke, K., \& Spitzer, R.L. (2002). The PHQ-9: a new depression diagnostic and severity measure. Psychiatric Annals, 32, 509-515.

Lamers, F., van Oppen, P., Smit, J. H., Spinhoven, P., van Balkom, A. J., Nolen, W. A.,.. Pennix, B. W. (2011). Comorbidity patterns of anxiety and depressive disorders in a large cohort study: the Netherlands Study of Depression and Anxiety (NESDA). Journal of Clinical Psychiatry,72, 341-348 
Launay, G., \& Slade, P. (1981). The measurement of hallucinatory predisposition in male and female prisoners. Personality and Individual Differences, 2, 221-234.

Lee, R. M., \& Robbins, S. B. (1998). The relationship between social connectedness and anxiety, self-esteem, and social identity. Journal of Counseling Psychology, 45, 338345.

MacLeod, C., Mathews, A., \& Tata, P. (1986). Attentional bias in emotional disorders. Journal of Abnormal Psychology, 95, 15-20.

Mathews, A., Ridgeway, V., \& Williamson, D.A. (1996). Evidence for attention to threatening stimuli in depression. Behaviour Research and Therapy, 34, 695-705.

McElroy, E., Fearon, P., Belsky, J., Fonagy, P., \& Patalay, P. (accepted, in press). The interconnectivity of depression and anxiety symptom networks through development. Journal of the American Academy of Child and Adolescent Psychiatry.

McElroy, E., \& Patalay., P. (under review). In search of disorders: Using network analysis to examine diagnostic boundaries in a large sample of help-seeking children.

McIntyre, J. C., Wickham, S., Barr, B., \& Bentall, R. P. (2017). Social identity and psychosis: Associations and psychological mechanisms. Schizophrenia Bulletin, 44, 681-690.

Melo, S., Corcoran, R., Shryane, N., \& Bentall, R.P. (2009). The persecution and deservedness scale. Psychology and Psychotherapy: Theory, Research and Practice, $82,247-260$.

Moffa, G., Catone, G., Kuipers, J., Kuipers, E., Freeman, D., Marwaha, S., ... \& Bebbington, P. (2017). Using directed acyclic graphs in epidemiological research in psychosis: an analysis of the role of bullying in psychosis. Schizophrenia Bulletin, 43, 1273-1279. 
Moutoussis, M., Williams, J., Dayan, P., \& Bentall, R.P. (2007). Persecutory delusions and the conditioned avoidance paradigm: towards an integration of the psychology and biology of paranoia. Cognitive Neuropsychiatry, 12, 495-510.

Nettle, D., Pepper, G.V., Jobling, R., \& Schroeder, K.B. (2014). Being there: a brief visit to a neighbourhood induces the social attitudes of that neighbourhood. PeerJ, 2, e236.

Opsahl, T., Agneessens, F., \& Skvoretz, J. (2010). Node centrality in weighted networks: Generalizing degree and shortest paths. Social Networks, 32, 245-251.

Peen, J., Schoevers, R.A., Beekman, A.T., \& Dekker, J. (2010). The current status of urbanrural differences in psychiatric disorders. Acta Psychiatrica Scandinavica, 121, 84-93.

Penkalla, A.M., \& Kohler, S. (2014). Urbanicity and mental health in Europe: a systematic review. European Journal of Mental Health, 9, 163-174.

Pons, P., \& Latapy, M. (2006). Computing communities in large networks using random walks. Journal of Graph Algorithms and Applications, 10, 191-218.

Roh, S., Jang, Y., Chiriboga, D. A., Kwag, K. H., Cho, S., \& Bernstein, K. (2011). Perceived neighborhood environment affecting physical and mental health: A study with Korean American older adults in New York City. Journal of Immigrant and Minority Health, 13,1005 .

Ross, C.E., \& Mirowsky, J. (2009). Neighborhood disorder, subjective alienation, and distress. Journal of health and Social Behavior, 50, 49-64.

Sampson, R.J., 2003. The neighborhood context of well-being. Perspectives in Biology and Medicine, 46, (Suppl. 3), S53-S64

Sani, F., Wakefield, J.R., Herrera, M., \& Zeybek, A. (2017). On the association between greater family identification and lower paranoid ideation among non-clinical individuals: evidence from Cypriot and Spanish students. Journal of Social and Clinical Psychology, 36, 396-418. 
Santos, H., Fried, E.I., Asafu-Adjei, J., \& Ruiz, R.J. (2017). Network structure of perinatal depressive symptoms in Latinas: relationship to stress and reproductive biomarkers. Research in Nursing \& Health, 40, 218-228.

Spitzer, R.L., Kroenke, K., Williams, J.B., \& Löwe, B. (2006). A brief measure for assessing generalized anxiety disorder: the GAD-7. Archives of Internal Medicine, 166, 10921097.

Stafford, M. A. I., Mcmunn, A., \& De Vogli, R. (2011). Neighbourhood social environment and depressive symptoms in mid-life and beyond. Ageing \& Society, 31, 893-910.

Sundquist, K., Frank, G., \& Sundquist, J. (2004). Urbanisation and incidence of psychosis and depression: follow-up study of 4.4 million women and men in Sweden. The British Journal of Psychiatry, 184, 293-298.

UK Cabinet Office. (2014). Community Life Survey questionnaire: 2013 to 2014. UK Cabinet Office.

UK Department for Communities and Local Government. (2015). The English Indices of Deprivation. 2015.

van Borkulo, C., Boschloo, L., Borsboom, D., Penninx, B.W., Waldorp, L.J., \& Schoevers, R.A. (2015). Association of symptom network structure with the course of depression. JAMA Psychiatry, 72. 1219-1226.

Van Borkulo, C. D., Borsboom, D., Epskamp, S., Blanken, T. F., Boschloo, L., Schoevers, R. A., \& Waldorp, L. J. (2014). A new method for constructing networks from binary data. Scientific Reports, 4, 5918.

Van Os, J., Linscott, R.J., Myin-Germeys, I., Delespaul, P., \& Krabbendam, L. (2009). A systematic review and meta-analysis of the psychosis continuum: evidence for a psychosis proneness-persistence-impairment model of psychotic disorder. Psychological Medicine, 39, 179-195. 
van Rooijen, G., Isvoranu, A. M., Meijer, C. J., van Borkulo, C. D., Ruhé, H. G., \& de Haan, L. (2017). A symptom network structure of the psychosis spectrum. Schizophrenia Research, 189, 75-83.

Vassos, E., Pedersen, C.B., Murray, R.M., Collier, D.A., \& Lewis, C.M. (2012). Metaanalysis of the association of urbanicity with schizophrenia. Schizophrenia Bulletin, $38,1118-1123$

Wickham, S., Taylor, P., Shevlin, M., \& Bentall, R. P. (2014). The impact of social deprivation on paranoia, hallucinations, mania and depression: the role of discrimination social support, stress and trust. PLoS One, 9, e105140.

Wilson-Genderson, M., \& Pruchno, R. (2013). Effects of neighborhood violence and perceptions of neighborhood safety on depressive symptoms of older adults. Social Science \& Medicine, 85, 43-49.

Witt, L.A. (1989). Urban-nonurban differences in social cognition: Locus of control and perceptions of a just world. The Journal of Social Psychology, 129, 715-717. 
Author contributions: E.M., J.M., R.P.B, and R.C. developed the study concept. All authors contributed to the study design. Data analysis was performed by E.M. E.M. J.M., R.P.B, and R.C. drafted the paper, and all authors provided critical revisions. All authors approved the final version of the paper for submission. 


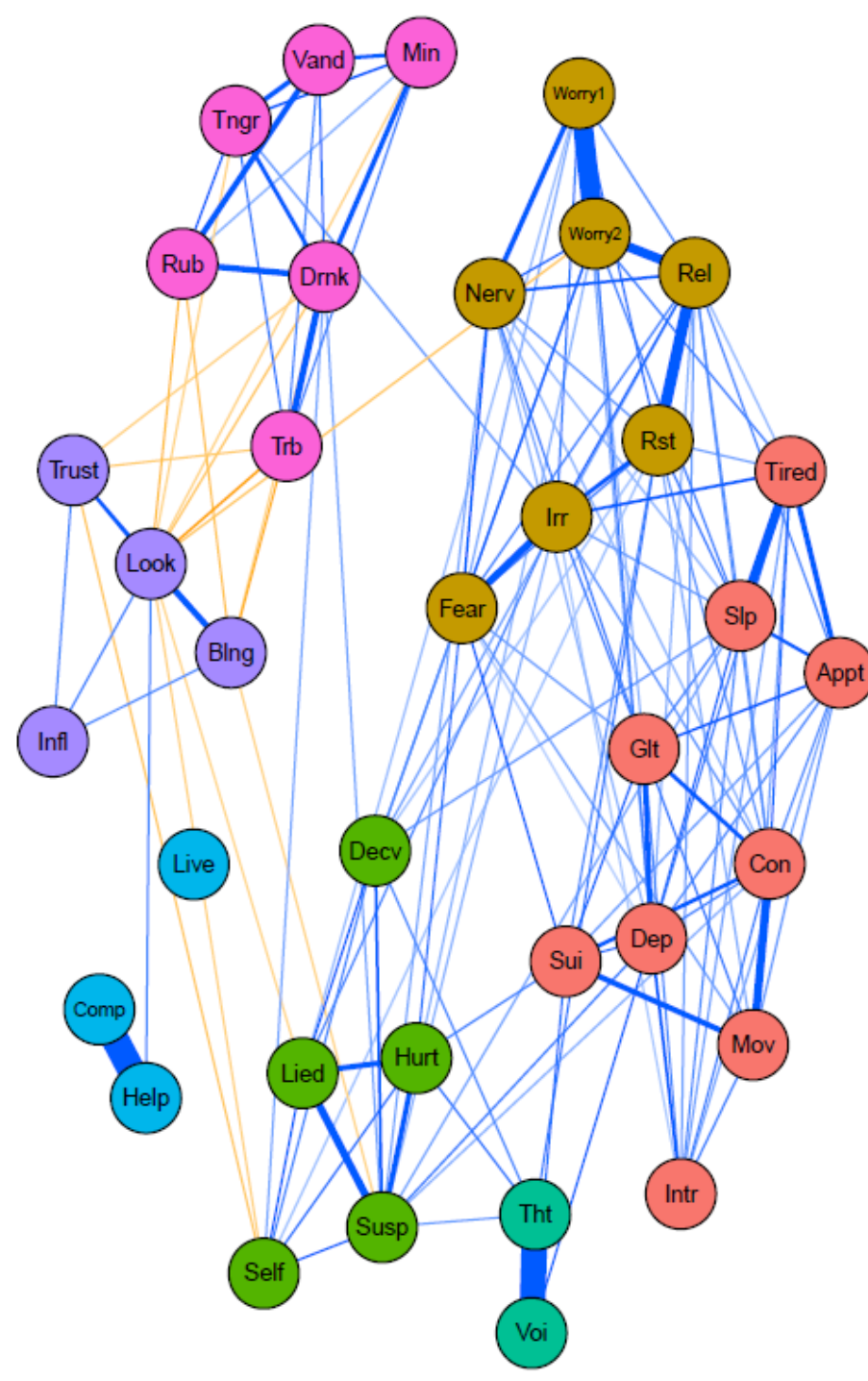

Depression

- Intr: Loss of interest

- Dep: Feeling down/depressed/hopeless

- Slp: Sleeping too much/little

- Tired: Feeling tired/lack energy

- Appt: Poor appetite or overeating

- Glt: Guilt

- Con: Concentration problems

- Mov: Moving/speaking too slow/fast

- Sui: Self-harm/suicidal thoughts

\section{General anxiety}

- Nerv: Feeling nervous, anxious or on edge

- Worry1: Can't control worry

- Worry2: Worrying too much about different things

- Rel: Trouble relaxing

- Rst: Feeling restless

- Irr: Easily annoyed or irritable

- Fear: Feeling afraid

\section{Paranoia}

- Decv: Worries about being deceived or harmed

- Susp: Suspicious of other people's intentions

- Lied: Feels that people will lie to them

- Hurt: Feels that people mean harm

Self: Only trusts self

\section{Auditory hallucinations}

- Voi: Hearing voices

- Tht: Voice speaks thoughts aloud

\section{Social connectivity}

- Live: Lives alone

- Help: Has people to call for help

- Comp: Has people to call for company

\section{Neighbourhood cohesion}

- Blng: Neighbourhood belonging

- Trust: Would give neighbour spare keys

- Look: Neighbours look out for each other

- Infl: Can influence local decisions

\section{Neighbourhood disorder}

- Drnk: People drunk/rowdy in public

- Rub: Rubbish/litter

- Vand: Vandalism

- Min: Harassment of minorities

- Tngr: Teenagers hanging around

- Trb: Troublesome neighbours

Figure 1. Elasso regularised network for overall sample $(\mathrm{N}=3670)$. Edges (lines) can be interpreted as partial correlations, with thickness denoting strength of effect. Blue lines reflect positive association, whereas red lines indicate negative association. 


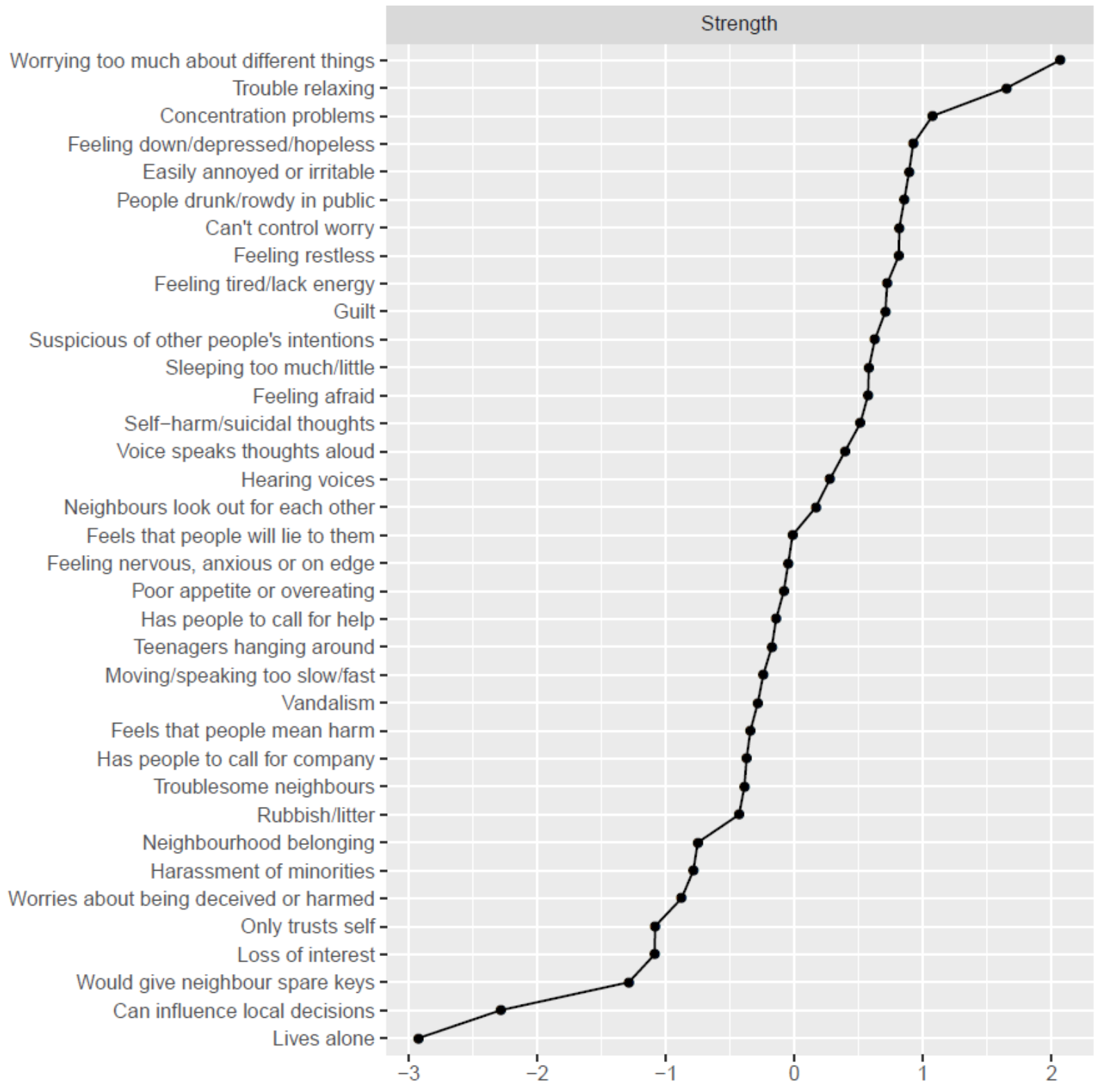

Figure 2. Centrality values presented as Z-scores. Higher values indicate greater overall importance to network. 
Loss of interest Feeling down/depressed/hopeless Sleeping too much/little -

Feeling tired/lack energy Poor appetite or overeating -

Guilt -

Concentration problems Moving/speaking too slow/fast Self-harm/suicidal thoughts Feeling nervous, anxious or on edgeCan't control worry Worrying too much about different things Trouble relaxing Feeling restless Easily annoyed or irritable Feeling afraid Worries about being deceived or harmed Suspicious of other people's intentions Feels that people will lie to them Feels that people mean harm Only trusts self Hearing voices Voice speaks thoughts aloud Lives alone -

Has people to call for help Has people to call for company Neighbourhood belonging Would give neighbour spare keys Neighbours look out for each other Can influence local decisions People drunk/rowdy in public Rubbish/litter Vandalism Harassment of minorities Teenagers hanging around Troublesome neighbours -

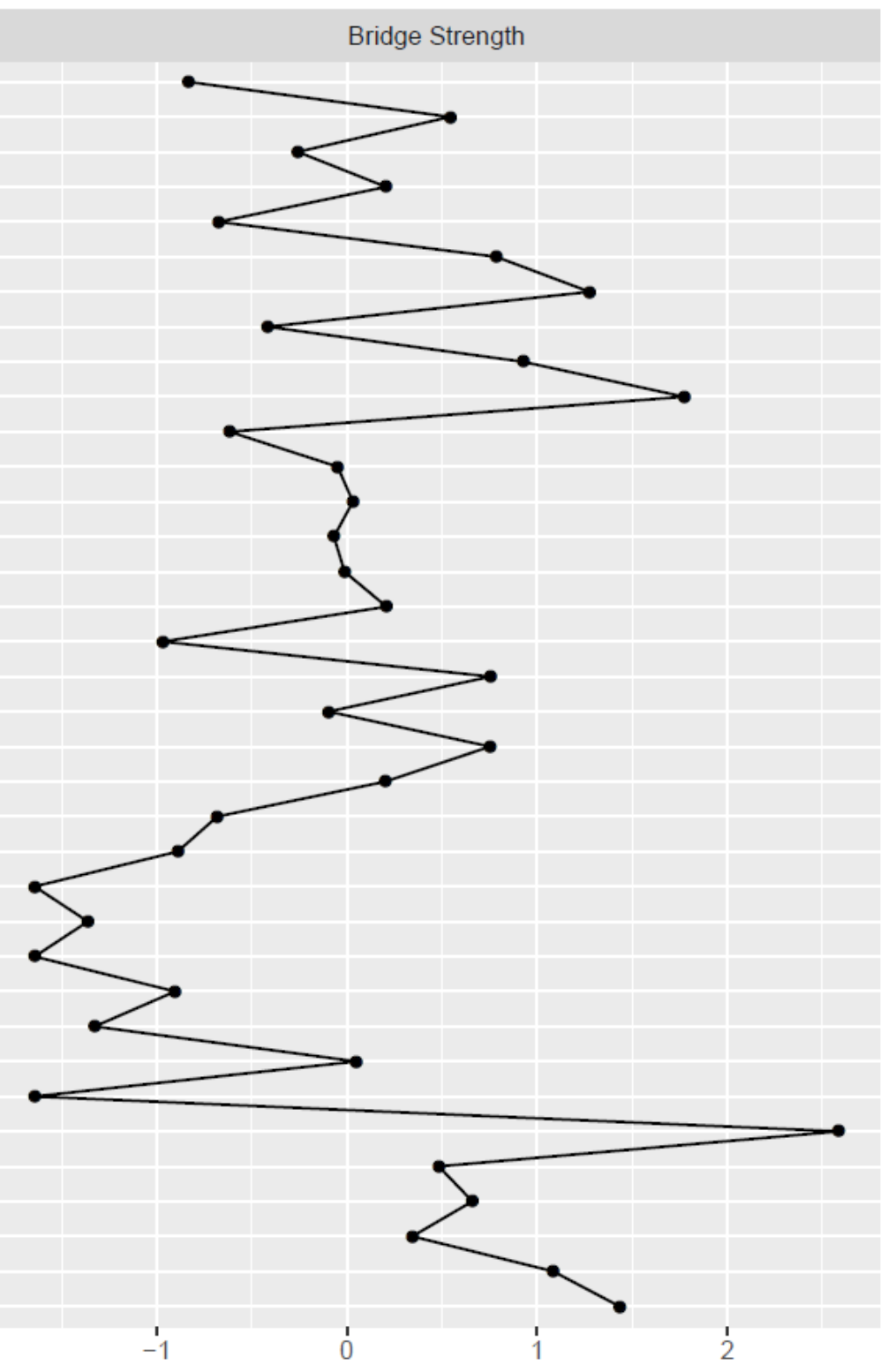

Figure 3. Bridge centrality values presented as Z-scores. Higher values indicate greater overall importance to network. 
Low Deprivation $(n=1,310)$

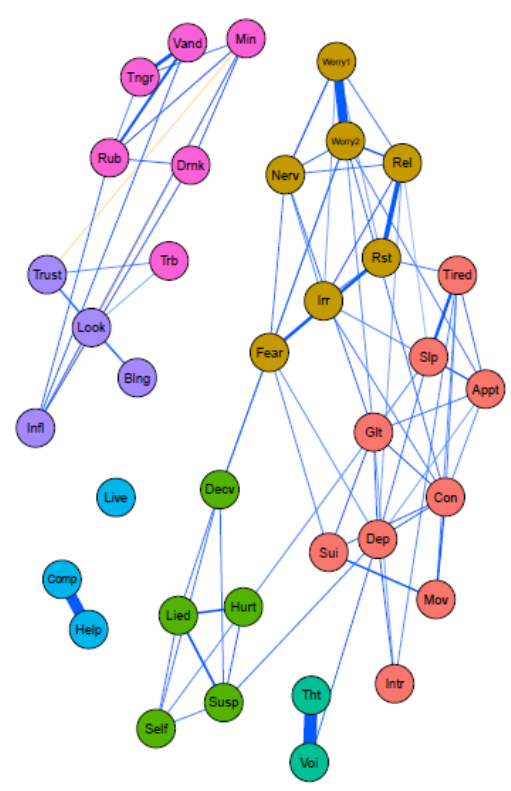

Moderate Deprivation $(n=1,192)$

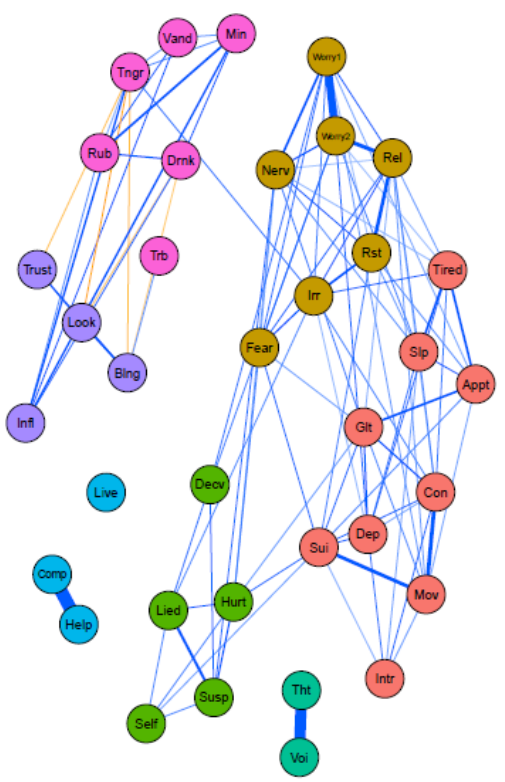

High Deprivation $(n=1,168)$

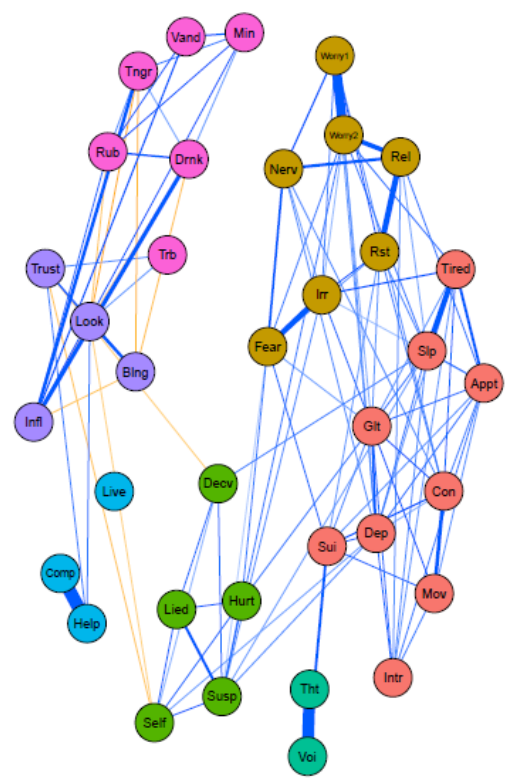

Figure 4. Elasso regularised network for the three separate deprivation groups. Edges (lines) can be interpreted as partial correlations, with thickness denoting strength of effect. Blue lines reflect positive association, whereas red lines indicate negative association. 